\title{
Phylogenetic Similarity of Popular Rice Varieties from Different Sources
}

Xavier Greg I Caguiat ${ }^{1 *}$ and Dindo A Tabanao ${ }^{2}$

${ }^{1}$ Genetic Resources Division, Philippine Rice Research Institute, Science City of Muñoz, Nueva Ecija 3119, Philippine

${ }^{2}$ Plant Breeding and Biotechnology Division, Philippine Rice Research Institute, Science City of Muñoz, Nueva Ecija 311, Philippine

\begin{abstract}
Superior rice varieties tend to be highly adopted by farmers across the country. Over time, samples of a variety coming from different places may exhibit some differences. Morphological descriptors are traditionally used to determine these differences however; these descriptors are limited in number and suffer from drawbacks such as influence of environment on trait expression and could not differentiate morphologically identical varieties. The highly reproducible molecular marker assay offers a powerful alternative to establish true identity and discriminate morphologically identical varieties. A study was conducted to determine the identity of several rice varieties based on DNA fingerprinting using genome-wide SSR markers. Samples of NSIC Rc240 from four sources, Aromatic Rice from three sources, and IR64 from two sources, were included in the study. Genetic similarity was calculated as proportion of shared alleles and cluster analysis was conducted using UPGMA. Results showed that the genetic similarity of three NSIC Rc240 samples was 1.0 confirming that these three samples were $100 \%$ genetically similar with each other while a fourth NSIC Rc240 sample from another source was only $89 \%$ similar to the other three. On the other hand, the three Aromatic Rice samples formed separate clusters at a range of $71-80 \%$ similarity. Lastly, a farmer's "improved" IR64 was only $59.5 \%$ similar to the original IR64. The observed divergence of samples with the same names in the study could be a result of further selection, gene flow, drift, admixture, or a combination of these mechanisms. This study underscores the importance of DNA fingerprinting analysis in variety identification, variation arising from selection and possible protection biopiracy.
\end{abstract}

Keywords: Divergence; DNA fingerprinting; Gene flow; Genetic drift

\section{Introduction}

Variety identification is an important aspect of the agriculture sector. The superiority of a variety over other available varieties in the market is mark through its proper identification. The name of the variety is accompanied by several rigorous processes that assure its uniqueness. This system could be summed up with the distinctness, uniformity and stability testing (DUST). Most often than not, these superior rice varieties tend to be highly adopted and patronized among farmers and consumers. Due to the popularity of such varieties in the market, other seed producer or grower would want to get a market share by means of 'branding' or labeling their products with the same variety name but at lower price than the original. When farmers bought from this fraud seller, they would found out that they have bought mislabeled products. The poor quality of the mislabeled product could suffice early during vegetative stage if the variation is on the morphology. However, if the mislabeled are almost similar to the original variety, farmers would spend ample time until harvest time before he could notice the discrepancy. Traditionally, morphological descriptors are routinely used for establishing the identity of varieties. But these morphological descriptors suffer from many drawbacks such as influence of environment on trait expression, epistatic interactions, pleiotropic effects etc. furthermore, the scarcity of sufficient number of these descriptors for unequivocal identification of increasing number of reference collection of varieties enforces to look for alternatives. Electrophoresis of seed proteins and isozyme analysis has overcome these limitations to some extent but now many powerful DNA based techniques are available [1].

The advent of molecular marker technology has revolutionized DNA-based molecular markers that could be utilized in various fields such as taxonomy, physiology, breeding, genetics, and plant genetic resources. Molecular markers are 'tags' that can be used to identify specific genes. These technologies will help ensure a desired beneficial trait is maintained or an undesirable trait eliminated. These 'tags' can also be used to identify a corresponding gene in a distant plant species or even in animal or microbial species. Advantages of DNA- based fingerprinting techniques includes, high-reproducibility, stage independent, only a small amount of plant tissue is needed, detection of pest and insect resistance even in the absence of the pest or insect, allows reliable tracking of beneficial traits, readily automated to increase cost efficiency, and the best way to establish differentiation for patent protection and Plant Variety Protection Act Certification and settlement of disputed varieties [2-4].

DNA fingerprinting has been widely used in the last two decades. It was widely used in genotype/cultivar identification in a wide range of crops species comprising cereals and pseudocereals (Echinochloa spp., Hordeum spp., Orzya spp., Secale cereal, Triticum spp., Zea mays), oilseeds (Arachis spp., Brassica spp., Glycine spp.), pulses (Cicer spp., Lens culinaris, Pisum sativum, Phaseolus spp., Vigna spp.), sugar yielding plants (Beta spp., Saccharum spp.), vegetables (Capsicum spp., Cucumis sativus, Lycopersicon esculentum, Solanum spp., Raphanus sativus) and fruits and nuts (Anacardium occidentale, Citrus spp., Mangifera indica, Malus spp., Musa spp., Prunus spp., Pyrus spp., Rubus spp., Vitis spp.) [5]. In rice, microsatellite or simple sequence repeat (SSR) markers are abundant and have been successfully exploited to develop superior rice cultivars tolerant to biotic (pest and disease resistance) and abiotic (drought, submergence tolerance, etc.) stresses. This marker system has also been established effective in DNA fingerprinting analysis to assess genetic relationships and to differentiate varieties from each other [2,6]. DNA fingerprinting can be used to provide proof of or defense against allegations of breach of

*Corresponding author: Xavier Greg I Caguiat, Senior Science Research Specialist, Genetic Resources Division 22 Philippine Rice Research Institute, Maligaya, Science City of Muñoz, Nueva Ecija 3119, Philippines, Tel: 09153710795 ; E-mail: xbieng03@gmail.com

Received July 04, 2016; Accepted July 29, 2016; Published August 05, 2016

Citation: Caguiat XGI, Tabanao DA (2016) Phylogenetic Similarity of Popular Rice Varieties from Different Sources. J Phylogenetics Evol Biol 4: 171. doi: 10.4172/2329-9002.1000171

Copyright: (c) 2016 Caguiat XGI, et al. This is an open-access article distributed under the terms of the Creative Commons Attribution License, which permits unrestricted use, distribution, and reproduction in any medium, provided the original author and source are credited. 
intellectual property rights. Such infringements would occur when 1) a registered variety is cultivated/marketed illegitimately under its own or a different name, 2) plant material comprising seeds, flowers, fruits or other plant products are falsely sold under the name of a registered variety, 3) plant material is collected from the wild and commercially exploited without authorization of biodiversity authority. Proof of infringement in all the above cases would require a rapid and unambiguous method of identification. A case of the use of molecular markers for resolving on IPR dispute was reported in India. The case relates to the unauthorized commercial sale of seeds of three spurious chili varieties marketed under the brand name of an elite variety. Fingerprinting results proved that the four chili samples were different from each other although that are being marketed under the name of one elite variety [7]. In India, similar case was reported on a special rice variety Aromatic Rice which is twice as expensive as normal rice in the European market. Adulteration of Aromatic Rice with large grain rice has frequently been reported. Until recently, determining a rice variety was limited to skilled visual inspection, combined with fragrance assessment, or sample grain measurements. DNA technology was applied to detect the presence of non-aromatic rice grains in samples of aromatic rice and quantify non-aromatic rice grains in a rice sample. A recent study by the British Food Standards Agency (FSA) using DNA technique revealed that of 363 samples collected from a range of outlets in UK, $17 \%$ contained over $20 \%$ conventional rice, and of these $9 \%$ contained more than $60 \%$ non-Aromatic Rice. European Union has recently imposed strict controls on Aromatic Rice quality including use of DNA-analysis [6].

Perez et al. [2] investigated the used of DNA analysis in detecting hybrid admixtures using microsatellite markers. They found out that DNAbased marker analysis can efficiently identify admixture in a short period of time compared with the conventional grow-out test [8,9]. Furthermore, minimum of two carefully chosen SSR markers could differentiate two different hybrid rice varieties while a single SSR marker could be used to differentiate hybrid seeds from non-hybrid seeds.

\section{Material and Methods}

\section{Plant materials}

Four important varieties were taken for this study which includes: Aromatic Rice, NSIC Rc240, NSIC Rc222 and IR64 and their respective counterparts. Samples of NSIC Rc240 from four sources, Aromatic Rice from three sources, and IR64 from two sources, were included in the study. Seeds of all genotypes were grown in field conditions at Philippine Rice Research Institute-Central Experimental Station, Muñoz, Nueva Ecija.

\section{DNA extraction}

Total DNA was extracted from fresh leaves with the use of Cetyl Tri-methyl Ammonium bromide (CTAB) method [10] with some modifications. The quality and concentration of DNA were estimated by using Nanodrop spectrophotometer (Thermo Scientific, Inc.). DNA was diluted in Tris-EDTA buffer for PCR analysis. The primer sequence information was obtained from publicly available sequence data [11].

\section{SSR analysis}

A total of 127 genome-wide SSR markers were used to verify the similarity of different rice varieties. DNA amplification was carried out in $4.1 \mu \mathrm{L}$ reaction volume containing $0.2 \mu \mathrm{M}$ of each primer, 20 $\mu \mathrm{M}$ of dNTPs, $1.5 \mu$ 10X PCR buffer, $1.5 \mathrm{mM} \mathrm{MgCl}_{2}, 50 \mathrm{ng}$ of DNA template and 0.5 unit of Taq DNA polymerase. The temperature profile used for the PCR amplification comprised of $94^{\circ} \mathrm{C}$ for $5 \mathrm{~min}$, followed by 35 cycles of $94^{\circ} \mathrm{C}$ for $1 \mathrm{~min}, 55^{\circ} \mathrm{C}$ for $1 \mathrm{~min}, 72^{\circ} \mathrm{C}$ for $2 \mathrm{~min}$ and ending up with $5 \mathrm{~min}$ at $72^{\circ} \mathrm{C}$ for the final extension. The annealing temperature was adjusted based on the specific requirements of each primer combination. The PCR products were electrophoresed in 5\% non-denaturing polyacrylamide gel at $100 \mathrm{~V}$ for $2 \mathrm{~h}$ (Figure 1). The gels were stained in ethidium bromide for $30 \mathrm{~min}$, de-stained for $15-30 \mathrm{~min}$ and then observed under a UV transilluminator.

Data gathered in the experiment was used as input data using Numerical Taxonomy Systems in personal computer (NTSYS-pc). The scores were standardized to unit variance using NTSYS program. Numerical measures of likeliness between each pair of accessions were conducted to produce a symmetrical square matrix which is necessary for classification techniques. NTYS-pc software was used to analyze these data. Similarity for Qualitative Data (SIMQUAL) was first computed to determine Jaccard's similarity coefficients. Principal components were used as input variables for cluster analysis using Unweighted Pair-Group Method with Arithmetic Averages (UPGMA). Using the 'Graphics' option, the computed UPGMA data were used to construct a dendrogram [12].

\section{Results and Discussion}

The application of DNA fingerprinting in elucidating variation and similarity of different popular rice in the Philippines could lead in a strong mechanism for variety protection and identification. It could be noted legal issues are also considered when it comes to commercialization. Thus, DNA fingerprinting is already admissible as substantial evidence in courts when it comes to biopiracy. The results highlighted here involve different cases when variation could arise and possible mechanism on how such variation arises.

\section{Selection pressure}

In the case of two sets of popular varieties: Aromatic Rice, a traditional variety, and NSIC Rc240, a released variety, showed variation due to intentional and unintentional selection. One case wherein three samples of Aromatic Rice were assayed with SSR markers. Genetic similarity was calculated as proportion of shared alleles from a marker dataset composed of 124 genomewide SSRs. The genetic similarity between the two checks, Aromatic Rice-A and Aromatic Rice-B, was only 0.80 (Table 1 ). Aromatic Rice A represents a sample that is fresh from the genebank, in other words, a sample of individuals that has not undergone recent selection yet. On the other hand, Aromatic Rice $\mathrm{B}$ has undergone many years of selection under Philippine conditions which could explain the significant amount of divergence from the

\begin{tabular}{|c|c|c|c|}
\hline & A & B & C \\
\hline A & 1.00 & - & - \\
\hline B & 0.8 & 1.00 & - \\
\hline C & 0.71 & 0.72 & 1.00 \\
\hline
\end{tabular}

Table 1: Genetic similarity between three samples of Aromatic Rice.
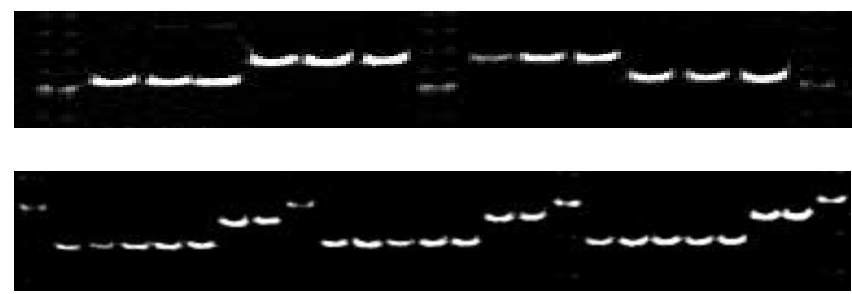

Figure 1: Representative gels of the polymorphic SSR markers in $5 \%$ nondenaturing polyacrylamide gel visualized in ethidium bromide. 
Aromatic Rice germplasm stored in the genebank. It could also be that the Aromatic Rice B originally came from a different accession of Aromatic Rice (i.e., not the same as the one from genebank). Aromatic Rice C is almost as genetically similar to Aromatic Rice B (0.72) as it is to Aromatic Rice A (0.71). This level of genetic similarity means that roughly $30 \%$ of the Aromatic Rice A genome is not the same as the Aromatic Rice B and Aromatic Rice A genomes, and could be considered as a significant amount of divergence. Indeed in Figure 2, the Aromatic Rice C is shown as an out group of the Aromatic Rice $\mathrm{A}$ and Aromatic Rice B cluster, implying that it is the most divergent among the three genotypes. Aromatic Rice $\mathrm{C}$ is said to have originated from Aromatic Rice B, so this divergence could mean a result of further selection, gene flow, drift, or more likely a combination of some or all three genetic forces. From experience, Aromatic Rice tends to outcross more than the average rice variety, causing some gene flow to occur which in turn provides variation for selection and drift to effect changes in allele frequencies. On the other hand, four samples of NSIC Rc240 from different sources were assayed using SSR markers. These were: (A) Nucleus seeds, (B) Foundation seeds, (C) Breeders' seeds and (D) Foundation seeds from another province. The genetic similarity among the first three NSIC Rc240 samples was 1.0 (Table 2). This means that these three samples are $100 \%$ genetically similar with each other. On the other hand, NSIC Rc240 from Isabela (D) is $89 \%$ similar to the three samples. Indeed in Figure 3, NSIC Rc240 from Isabela is shown as an out group of the other three, implying that it is the most divergent among the four genotypes.

\section{Labeling versus branding}

The second set of materials used in the study includes high-yielding varieties NSIC Rc222 (versus Diamond X) and mega variety IR 64 (versus "improved" IR64) which are compared against their supposed counterpart. It could be noted that through molecular analysis that the counterpart are not $100 \%$ similar to the original. In case of NSIC Rc222 as compared with a supposed brand "Diamond X" with three replicates each were assayed using SSR markers.

The genetic similarity between replicates was 1.0 for both NSIC Rc222 and Diamond X but differs as much as $32 \%$ between the two samples (Table 3). This means that these two samples are not $100 \%$ genetically similar with each other (Figure 4 ). On the other hand, IR64 was compared with a supposed "Improved IR64" with

\begin{tabular}{|c|c|c|c|c|}
\hline & $\begin{array}{c}\text { NSIC Rc240 } \\
\text { A }\end{array}$ & $\begin{array}{c}\text { NSIC Rc240 } \\
\text { B }\end{array}$ & $\begin{array}{c}\text { NSIC Rc240 } \\
\text { C }\end{array}$ & $\begin{array}{c}\text { NSIC Rc240 } \\
\text { D }\end{array}$ \\
\hline NSIC Rc240 A & 1.00 & - & - & - \\
\hline NSIC Rc240 B & 1.00 & 1.00 & - & - \\
\hline NSIC Rc240 C & 1.00 & 1.00 & 1.00 & - \\
\hline NSIC Rc240 D & 0.89 & 0.89 & 0.89 & 1.00 \\
\hline
\end{tabular}

Table 2: Genetic similarity among the four samples of NSIC Rc240.

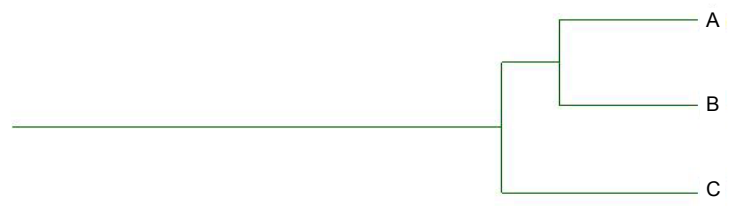

$0.00 \quad \begin{gathered}0.50 \\ 0.25 \quad 0.75\end{gathered}$

Figure 2: UPGMA dendrogram of three samples of Aromatic Rice 370 based on genetic similarity expressed as proportion of shared alleles.

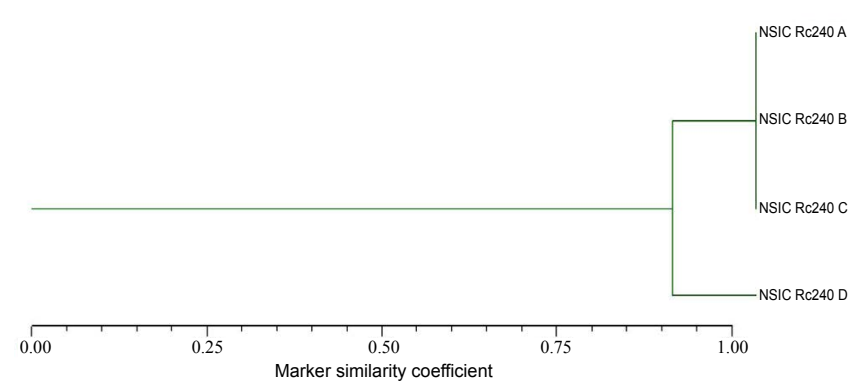

Figure 3: UPGMA dendrogram of four samples of NSIC Rc240 based on genetic similarity expressed as proportion of shared alleles.
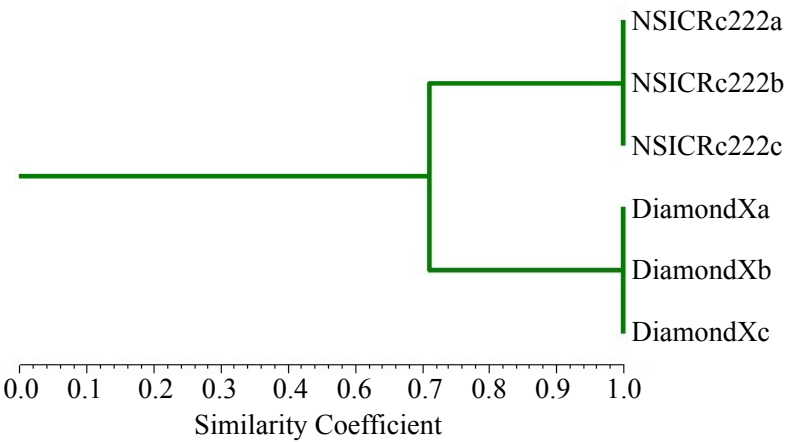

Figure 4: UPGMA dendrogram of three replicate samples of NSIC Rc222 and Diamond $X$ based on genetic similarity expressed as proportion of shared alleles.

three replicates each were assayed using SSR markers. The genetic similarity between replicates was 1.0 for both IR64 and "Improved IR64" but differs as much as $37 \%$ between the two samples (Table 4). This means that these two samples are not genetically similar with each other (Figure 5).

It is of great importance to know that certain varieties are being commercialized due to their advantages over other available varieties. These advantages include resistance to biotic stresses, tolerance to abiotic stresses and higher yield. There were several instances that legal cases were filed due to branding or unauthorized commercialization of certain variety. One instance is a case of marketing of spurious seeds of chilli, Capsicum annum in the brand name of an elite variety using inter simple sequence repeat polymerase chain reaction (ISSR-PCR) and FISSR-PCR (for fluorescent ISSR-PCR) which found that there was really a violation of Plant Breeder's Rights [13]. One pioneering study in eggplant diversity in the Philippines had used morphological, molecular and combination of both analyses had resulted to findings that some variety are exactly the same in term of morphological and molecular analyses and that difference in naming maybe a result of marketing advantage $[14,15]$. In rice, the most popular case was between India and US Company wherein Basmati rice lines was the center of the dispute. The case filed by India against the company who applied for patent of almost the same rice lines where based on three things: theft involved in the Basmati patent is, therefore, threefold: a theft of collective intellectual and biodiversity heritage on Indian farmers, a theft from Indian traders and exporters whose markets are being stolen by the US Company, and finally a deception of consumers since name Basmati rice was stole which are derived from Indian rice but not grown in India, and hence are not the same quality (http:// www1.american.edu/) [16]. 
Citation: Caguiat XGI, Tabanao DA (2016) Phylogenetic Similarity of Popular Rice Varieties from Different Sources. J Phylogenetics Evol Biol 4: 171. doi: 10.4172/2329-9002.1000171

Page 4 of 4

\begin{tabular}{|c|c|c|c|c|c|}
\hline & NSICRc222a & NSICRc222b & NSICRc222c & DiamondXa & DiamondXb \\
\hline NSICRc222a & 1.00 & - & - & - \\
\hline NSICRc222b & 1.00 & 1.00 & - & - \\
\hline NSICRc222c & 1.00 & 1.00 & 1.00 & - & - \\
\hline DiamondXa & 0.68 & 0.68 & 0.68 & - \\
\hline DiamondXb & 0.68 & 0.68 & 0.68 & - & - \\
\hline DiamondXc & 0.68 & 0.68 & 0.68 & 1.00 & - \\
\hline
\end{tabular}

Table 3: Genetic similarity among the samples of NSIC Rc222 and Diamond X.

\begin{tabular}{|c|c|c|c|c|c|c|}
\hline & IR64a & IR64b & IR64c & ImprovedIR64a & ImprovedIR64b & ImprovedIR64c \\
\hline IR64a & 1.00 & - & - & - & - & - \\
\hline IR64b & 1.00 & 1.00 & - & - & - & - \\
\hline IR64c & 1.00 & 1.00 & 1.00 & - & - & - \\
\hline ImprovedIR64a & 0.63 & 0.63 & 0.63 & 1.00 & - & - \\
\hline ImprovedIR64b & 0.63 & 0.63 & 0.63 & 1.00 & 1.00 & - \\
\hline ImprovedIR64c & 0.63 & 0.63 & 0.63 & 1.00 & 1.00 & 1.00 \\
\hline
\end{tabular}

Table 4: Genetic similarity among samples of IR64 and "Improved IR64".

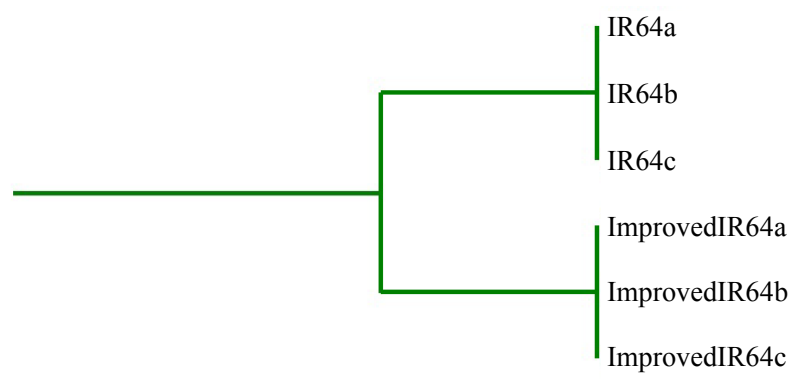

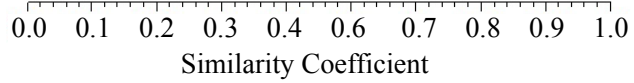

Figure 5: UPGMA dendrogram of three replicate samples of IR64 versus "Improved IR64" based on genetic similarity expressed as proportion of shared alleles.

\section{Conclusion}

The genetic similarity of three NSIC Rc240 samples was 1.0 confirming that these three samples were $100 \%$ genetically similar with each other while a fourth NSIC Rc240 sample from another source was only $89 \%$ similar to the other three. On the other hand, the three Aromatic Rice samples formed significantly separate clusters at a range of $71-80 \%$ similarity. Lastly, a farmer's “improved" IR64 was only $59.5 \%$ similar to the original IR64 and NSIC Rc222 was $68 \%$ similar to Diamond X. The observed divergence of samples with the same names in the study could be a result of further selection, gene flow, drift, admixture, or a combination of these mechanisms while the supposed similar variety being commercialized in different name could be an essentially derived variety. This study underscores the importance of DNA fingerprinting in variety identification and protection of Breeder's rights.

\section{Conflict of Interest}

The authors declare no conflict of interest in this paper. All authors are company employees as stated in the affiliations. The authors received no additional financial gain from this publication.

\section{References}

1. Henry RJ (2001) (ed.) Plant genotyping-the DNA fingerprinting of plants. CABI Publishing, UK.

2. Perez LM, Pastor HM, Domingo JM, Tabanao DA, Manigbas NL (2012) DNA fingerprinting in hybrid rice. Philippine Rice Research Institute, Nueva Ecija.
3. Tamilkumar P, Jerlin R, Senthil N, Ganesan KN, Jeevan RJ, et al. (2009) Fingerprinting of rice hybrids and their parental lines using microsatellite markers and their utilization in genetic purity assessment of hybrid rice. Research Journal of Seed Science 2: 40-47.

4. Jeffreys AJ, Wilson V, Thein SL (1985) Individual specific fingerprints of human DNA. Nature 316: 76-79.

5. Heckenberger M, Bohn M, Ziegle JS, Joe LK, Hauser JD, et al. (2002) Variation of DNA fingerprints among accessions within maize inbred lines and implications for identification of essentially derived varieties. I. Genetic and technical sources of variation in SSR data. Mol Breed 10: 181-191.

6. Karp A, Edwards KJ (1997) Molecular techniques in the analysis of the extent and distribution of genetic diversity.

7. Ayad WG, Hodgkin T, Jaradat A, Rao VR (1995) Molecular genetic techniques for plant genetic resources. Report of an IPGRI Workshop, International Plant Genetic Resources Institute, Rome, pp: 11-38.

8. Kumar LD, Kathirvel M, Rao GV, Nagaraju J (2000) DNA profiling of disputed chili samples (Capsicum annum) using ISSR=PCR and FISSR-PCR marker assay. Forensic Sci Int 116: 63-68.

9. Food Standards Agency (2004) Survey on Aromatic Rice.

10. Moorthy KK, Babu P, Sreedhar M, Sama VSAK, kumar PN, et al. (2011) Identification of informative EST-SSR markers capable of distinguishing popular Indian rice varieties and their utilization in seed genetic purity assessments. Seed Science and Technology 39: 282-292.

11. Naresh V, Yamini KN, Rajendrakumar P, Kumar VD (2009) EST-SSR markerbased assay for the genetic purity assessment of safflower hybrids. Euphytica 170: 3470353

12. Murray HG, Thompson WF (1980) Rapid isolation of high molecular weight DNA. Nucleic Acids Res 8: 4321-4325.

13. www.gramene.org

14. Rohlf FJ (2000) NTSYS-pc: Numerical Taxonomy and Multivariate Analysis System, Version 2.1. Exeter Software, Setauket, NY.

15. Caguiat XGI, Hautea DM (2014) Genetic diversity analysis of eggplant (Solanum melongena $L$.) and related wild species in the Philippines using morphologica and SSR markers. SABRAO Journal of Breeding and Genetics 46: 183-201.

16. TED Case Studies. 